\title{
ChemComm
}

Cite this: Chem. Commun., 2013, 49, 4652

Received 25th February 2013, Accepted 2nd April 2013

DOI: $10.1039 / \mathrm{c3cc4} 1438 \mathrm{~h}$

\section{Polymerase synthesis of oligonucleotides containing a single chemically modified nucleobase for site-specific redox labelling $\dagger$}

\author{
Petra Ménová, ${ }^{a}$ Hana Cahová, ${ }^{a}$ Medard Plucnara, ${ }^{\text {b }}$ Luděk Havran, ${ }^{\text {b }}$ \\ Miroslav Fojta*bc and Michal Hocek*ad
}

www.rsc.org/chemcomm

Enzymatic construction of single-nucleobase redox-labelled oligonucleotides was developed either based on polymerase incorporation of a single modified nucleoside triphosphate (dNTP) followed by primer extension (PEX) with natural dNTPs or based on PEX with a biotinylated one-nucleotide overhang template, magnetoseparation and the second PEX with a full-length template.

Enzymatic synthesis of base-modified DNA and oligonucleotides (ONs) by polymerase incorporation of modified nucleoside triphosphates (dNTPs) is now an established method ${ }^{1}$ often used for fluorescence, ${ }^{2}$ redox, ${ }^{3}$ spin $^{4}{ }^{4}$ barcode $^{5}$ and reactive ${ }^{6}$ labelling, as well as for the protection of DNA. ${ }^{7}$ Primer extension (PEX) is the best approach for the synthesis of medium-sized ONs bearing several modifications, Nicking Enzyme Amplification Reaction is used for short single stranded ONs (ssONs), ${ }^{8}$ terminal transferase incorporation for $3^{\prime}$-tail labelling, ${ }^{9}$ whereas PCR is used for the synthesis of long DNA duplexes containing a large number of modifications. The PEX method can be combined with magnetoseparation on streptavidin ${ }^{3}$ beads to produce ssONs.

However, in all these methods, the modified dNTP is used instead of one of the natural dNTPs and, therefore, in all the newly synthesized stretches, all the copies of this particular nucleobase are fully modified. None of these methods is suitable for the introduction of a single base-modification at a specific internal position within the sequence. However, specifically single-labelled ON probes are needed for most

\footnotetext{
${ }^{a}$ Institute of Organic Chemistry and Biochemistry, Academy of Sciences of the Czech Republic, Gilead \& IOCB Research Center, Flemingovo nam. 2, CZ-16610 Prague 6, Czech Republic.E-mail: hocek@uochb.cas.cz

${ }^{b}$ Institute of Biophysics, v.v.i. Academy of Sciences of the Czech Republic, Kralovopolska 135, 61265 Brno, Czech Republic.E-mail: fojta@ibp.cz ${ }^{c}$ Central European Institute of Technology, Masaryk University, Kamenice 753/5, CZ-625 00 Brno, Czech Republic

${ }^{d}$ Department of Organic and Nuclear Chemistry, Faculty of Science, Charles University in Prague, Hlavova 8, CZ-12843 Prague 2, Czech Republic

$\dagger$ Electronic supplementary information (ESI) available: Additional examples of monoincorporation of other dNTPs into other sequences, additional figures, experimental procedures, MALDI spectra and melting temperature curves. See DOI: $10.1039 / \mathrm{c} 3 \mathrm{cc} 41438 \mathrm{~h}$
}

bioanalytical applications and that is why we report here a new general enzymatic methodology to prepare them.

The methodology was developed and tested for the synthesis of ssON probes bearing a single redox label ${ }^{3}$ - either an electroreducible 3-nitrophenyl group or electrooxidizable 3 -aminophenyl ${ }^{3 b}$ and ferrocenyl groups. ${ }^{3 a}$ Therefore, nitrophenyl- (dN $\left.{ }^{\mathbf{N O} 2} \mathbf{T P s}\right)$, aminophenyl- ( $\left.\mathbf{d N}^{\mathbf{N H 2}} \mathbf{T P s}\right)$ and ferrocenylethynyl-modified dNTPs $\left(\mathbf{d N}^{\mathbf{F c}} \mathbf{T P s} \text {, Chart } 1\right)^{3 a, b, 9}$ derived from all four nucleobases were tested and used as substrates for polymerase incorporation. The modified $\mathbf{d G}^{\mathbf{X}} \mathbf{T P s}$ were prepared according to improved methods (see ESI $\dagger$ ). Vent(exo-), Pwo and KOD XL were tested as enzymes in this methodology. For the list of sequences used in this study, see Table S1 in ESI. $†$

The plan was to perform single-nucleotide incorporation (SNI) of a modified $\mathbf{d} \mathbf{N}^{\mathbf{X}}$ TP followed by PEX using natural dNTPs (Scheme 1a). Therefore, the first task was to optimize the SNI with each of the 12 modified $\mathbf{d} \mathbf{N}^{\mathbf{X}}$ TPs to get full conversion to a one-nucleotide longer primer with possibly minimum excess of the modified $\mathbf{d} \mathbf{N}^{\mathbf{X}} \mathbf{T P}$ (to prevent its further incorporation during the PEX). After initial optimization, Vent(exo-) was selected as the best enzyme (Pwo was found to digest the template in the absence of dNTPs due to exonuclease activity and KOD XL did not give clean SNI products). Then, general conditions for SNI

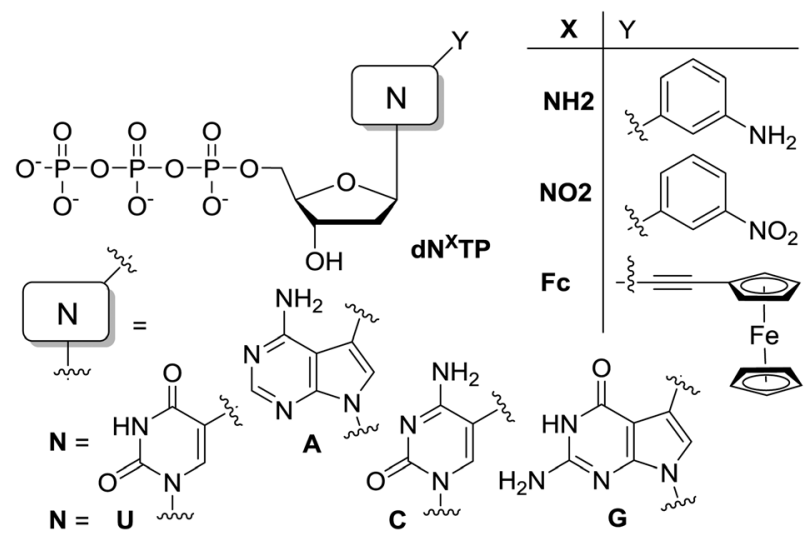

Chart 1 Structures of modified $\mathbf{d N}^{\mathrm{X}}$ TPs used in this study. 


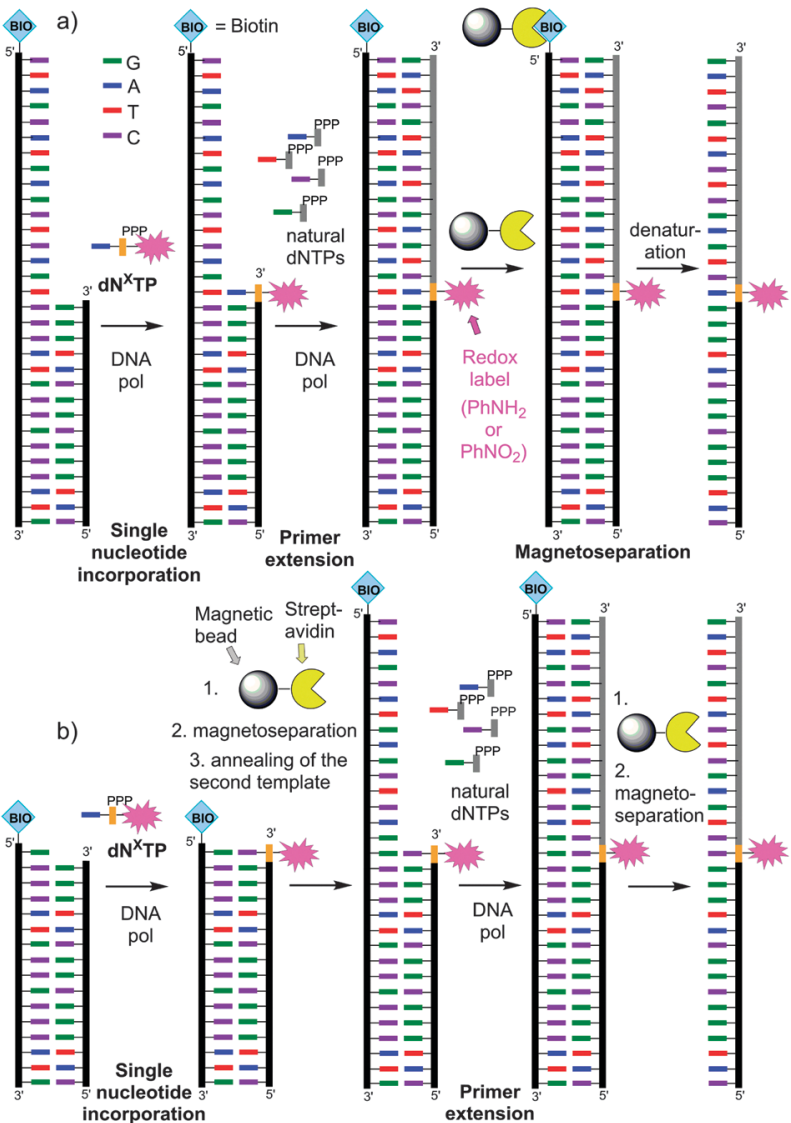

Scheme 1 Outline of the synthesis of ssON containing a single modification (a) followed by a different nucleobase, (b) followed by the same but unmodified nucleobase.

applicable to any of the 12 modified $\mathbf{d} \mathbf{N}^{\mathbf{X}}$ TPs (freshly dissolved solutions should be used) were found when using 1.3 equiv. of $\mathbf{d N}^{\mathbf{X}} \mathbf{T P}$ at $60{ }^{\circ} \mathrm{C}$ for $5 \mathrm{~min}$ to obtain in all cases clean products extended by one-nucleotide (Fig. 1a and b and Fig. S1-S4 in ESI $\dagger$ - lanes 3). For SNI of modified $\mathbf{d} \mathbf{A}^{\mathbf{X}}$ TPs (which are known ${ }^{3}$ to be the best substrates for Vent(exo-) polymerase), much lower concentrations of the polymerase $\left(0.004\right.$ or $0.02 \mathrm{U}_{\mu} \mathrm{L}^{-1}$ for $\mathbf{d A} \mathbf{A}^{\mathbf{N H 2}} \mathbf{T P}$ or $\mathbf{d} \mathbf{A}^{\mathbf{N O 2}} \mathbf{T P}$, respectively) were used than with all other $\mathbf{d N}^{\mathbf{X}} \mathbf{T P s}\left(0.1 \mathrm{U} \mu \mathrm{L}^{-1}\right)$. After the completion of the SNI, the mix of all four natural dNTPs (1000 equiv. each) was added and the polymerase performed the PEX for 20 min to give full-length products (lanes 6 in all gels). When the procedure was performed on a biotinylated template, the final magnetoseparation (Scheme 1a) furnished the desired pure single-point modified sSON which was characterized using MALDI-TOF (see Table S2 in ESI $\dagger$ ).

This simple SNI-PEX methodology is generally applicable for the incorporation of a single modification into all sequences when the modified nucleotide is followed by a different nucleobase. If the same nucleobase should be present next to the modification, the polymerase would tend to incorporate the second modified nucleotide (even at low concentrations of $\mathbf{d N}^{\mathbf{X}} \mathbf{T P}$ ). Therefore a modified procedure had to be developed for such sequences (Scheme 1b). It consists of the SNI using a biotinylated template only one-nucleotide longer than the

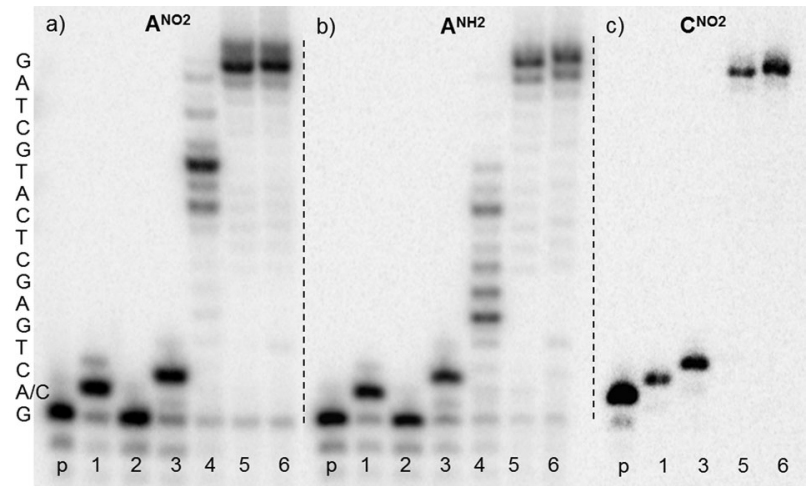

Fig. 1 Denaturing PAGE analysis of products of polymerase synthesis of ONs bearing a single modification either by SNI-PEX (a) and (b) $\left(A^{\mathrm{NO2}}\right.$ or $\left.A^{\mathrm{NH} 2}\right)$ or (c) by an indirect SNI-magnetoseparation-PEX approach $\left(\mathrm{C}^{\mathrm{NO} 2}\right)$. Experiments are supplemented with a primer (p). Lane 1: positive control for SNI (either dATP or dCTP); lane 2: negative control for SNI (no dNTPs present); lane 3: SNI of $\mathbf{d N}^{\mathbf{X}}$ (only $\mathbf{d N}^{\mathrm{X}}$ TP present); lane 4: negative control for PEX (absence of either dATP or dCTP); lane 5: positive control for PEX (all natural dNTPs present); lane 6: PEX of SNI-product containing $\mathbf{d N}^{\mathrm{X}}$ (all natural dNTPs present). In the case of a sequence when the modification ( $c$ ) $\left(\mathrm{C}^{\mathrm{NO}}\right)$ is followed by the same unmodified nucleotide, the magnetoseparation of the SNI-product (lane 3) was followed by the PEX using a longer template to give the final modified ON (lane 6).

primer (to prevent additional incorporation of the second modified $\mathbf{d N}^{\mathbf{X}} \mathbf{T P}$ ), magnetoseparation of the extended primer, annealing with the full-length template and the final PEX (followed by magnetoseparation in preparative experiments). Fig. 1c (and Fig. S5 in ESI $\dagger$ ) shows that this procedure gives clean modified ssON products (which were also isolated by magnetoseparation and characterized using MALDI).

Once having all the 4 modified ONs (ON1-ON4 containing one $\mathbf{d N}^{\mathbf{N O 2}}$ ) in hands, we studied the influence of the modification on the denaturation temperature $\left(T_{\mathrm{m}}\right)$. Table S3 (ESI $\dagger$ ) shows that the nitrophenyl modification destabilizes the duplexes $\left(\Delta T_{\mathrm{m}}=-0.1\right.$ to $-2.2{ }^{\circ} \mathrm{C}$ depending on the base-pair).

For any applications of redox labelling in DNA diagnostics, we need to know the sequence dependence of the redox potential. If the sequence dependence is very high, the apparent redox potential could directly give information about an unknown sequence. On the other hand, for multi-potential labelling $^{3 c, d}$ of each nucleobase with a different label, the requirement is to have the possibly narrowest dispersion of redox potentials so that the response is additive and the potentials of different labels cannot interfere.

In order to study electrochemical properties of the monoincorporation products including the sequence dependence, we have prepared a set of ssONs containing either of the four $\mathbf{d N}^{\mathbf{N O 2}}$ labels in sequence contexts (Table 1). This also confirms that the methodology is truly general in terms of the sequence of the target ON. The nitro-label was chosen with respect to a high electron yield of its electroreduction (4 electrons per label under the given conditions), ${ }^{3 b}$ giving rise to a well measurable signal even for a single redox tag among several tens of unmodified nucleotides (Fig. S8 in ESI $\dagger$ ). To attain good reproducibility we performed the measurements using a hanging mercury drop electrode. As it is evident from Table 1, the apparent potentials of the nitro label primary reduction 
Table 1 Potentials of $\mathrm{N}^{\mathrm{NO} 2}$ reduction in different sequence contexts

\begin{tabular}{llll}
\hline & \multicolumn{2}{l}{$E^{a} / \mathrm{V}$} & \\
\hline AAA & -0.505 & TUC & $E^{a} / \mathrm{V}$ \\
CAA & -0.505 & GUC & -0.510 \\
CAC & -0.505 & GUA & -0.515 \\
CAT & -0.505 & TCT & -0.505 \\
GAA & -0.500 & GCT & -0.495 \\
GAC & -0.490 & GCA & -0.510 \\
GAG & -0.485 & TGC & -0.515 \\
GAT & -0.485 & GGC & -0.510 \\
TAA & -0.500 & GGA & -0.515 \\
TAT & -0.490 & & -0.505 \\
& & &
\end{tabular}

${ }^{a}$ Peak potentials at the hanging mercury drop electrode against $\mathrm{Ag}|\mathrm{AgCl}| 3 \mathrm{M} \mathrm{KCl}$. The nitrophenyl-modified bases are always in the center of the triplet (in bold); other parts of the ONs outside these triplets are identical in all of them (see ON1-ON23 sequences in Table S1). For more details see the ESI.

depended to some extent on both the conjugate nucleobases and the sequence context. However, especially variations in the peak potentials reflecting changes in the sequence context were relatively small, in most cases at the preciseness limit of the reference electrode and the instrument setup $(5-10 \mathrm{mV})$. It seems that the presence of thymine and/or guanine next to the modification site had the most pronounced effect on the peak potential, shifting the nitro group reduction to less negative values (Table 1).

In conclusion, we have developed the first general methodology for enzymatic synthesis of ssONs bearing a single basemodification in the internal part of the sequence. A simple and facile SNI-PEX (+magnetoseparation) procedure is applicable to all sequences when the modification is followed by another nucleobase. If the modification needs to be incorporated into a homonucleobase stretch, the SNI is performed using a biotinylated template one-nucleotide longer than the primer and after the magnetoseparation the extended primer is subjected to another PEX with a full-length template. This procedure is more laborious but is generally applicable to any sequence. This novel methodology enables for the first time preparation of ssON probes bearing one site specific single modification (i.e. redox or other label) at one nucleobase using enzymatic synthesis. The only limitation is that the $\mathbf{d N} \mathbf{N}^{\mathbf{X}} \mathbf{T P}$ must be a good substrate for DNA polymerase. The approach is likely to find broad applications in the construction of diverse base-modified ON probes. We have demonstrated its utility by the synthesis of different labelled sequences (containing one $\mathbf{d N}^{\mathbf{N O 2}}$ nucleotide) which showed that the influence of the sequence on the redox potential is relatively low (max. $10 \mathrm{mV}$ differences). These data support our on-going project of multi-potential redox labelling for electrochemical detection. ${ }^{3 c, d}$

This work was supported by the Academy of Sciences of the Czech Republic (RVO: 61388963 and 68081707) and by the Czech Science Foundation (P206-12-G151).

\section{Notes and references}

1 (a) M. Hocek and M. Fojta, Org. Biomol. Chem., 2008, 6, 2233-2241; (b) S. Jäger, G. Rasched, H. Kornreich-Leshem, M. Engeser, O. Thum and M. Famulok, J. Am. Chem. Soc., 2005, 127, 15071-15082; (c) P. Čapek, H. Cahová, R. Pohl, M. Hocek, C. Gloeckner and A. Marx, Chem.-Eur. J., 2007, 13, 6196-6203.

2 (a) L. H. Thoresen, G.-S. Jiao, W. C. Haaland, M. L. Metzker and K. Burgess, Chem.-Eur. J., 2003, 9, 4603-4610; (b) J. Riedl, R. Pohl, L. Rulíšek and M. Hocek, J. Org. Chem., 2012, 77, 1026-1044; (c) J. Riedl, R. Pohl, N. P. Ernsting, P. Orsag, M. Fojta and M. Hocek, Chem. Sci., 2012, 3, 2797-2806; (d) J. Riedl, P. Ménová, R. Pohl, P. Orság, M. Fojta and M. Hocek, J. Org. Chem., 2012, 77, 8287-8293.

3 (a) P. Brázdilová, M. Vrábel, R. Pohl, H. Pivoňková, L. Havran, M. Hocek and M. Fojta, Chem.-Eur. J., 2007, 13, 9527-9533; (b) H. Cahová, L. Havran, P. Brázdilová, H. Pivoňková, R. Pohl, M. Fojta and M. Hocek, Angew. Chem., Int. Ed., 2008, 47, 2059-2062; (c) M. Vrábel, P. Horáková, H. Pivoňková, L. Kalachova, H. Černocká, H. Cahová, R. Pohl, P. Šebest, L. Havran, M. Hocek and M. Fojta, Chem.-Eur. J., 2009, 15, 1144-1154; (d) J. Balintová, R. Pohl, P. Horáková, P. Vidláková, L. Havran, M. Fojta and M. Hocek, Chem.-Eur. J., 2011, 17, 14063-14073; (e) V. Raindlová, R. Pohl, B. Klepetářová, L. Havran, E. Šimková, P. Horáková, H. Pivoňková, M. Fojta and M. Hocek, ChemPlusChem, 2012, 77, 652-662; $(f)$ D. A. Di Giusto, W. A. Wlassoff, S. Geisebrecht, J. J. Gooding and G. C. King, J. Am. Chem. Soc., 2004, 126, 4120-4121; $(g)$ D. A. Di Giusto, W. A. Wlassoff, S. Geisebrecht, J. J. Gooding and G. C. King, Angew. Chem., Int. Ed., 2004, 43, 2809-2812; (h) W. A. Wlassoff and G. C. King, Nucleic Acids Res., 2002, 30, e58; (i) S. S. W. Yeung, T. M. H. Lee and I.-M. Hsing, J. Am. Chem. Soc., 2006, 128, 13374-13375; $(j)$ H. Weizman and Y. Tor, J. Am. Chem. Soc., 2002, 124, 1568-1569.

4 S. Obeid, M. Yulikow, G. Jeschke and A. Marx, Angew. Chem., Int. Ed., 2008, 47, 6782-6785.

5 A. Baccaro, A.-L. Steck and A. Marx, Angew. Chem., Int. Ed., 2012, 51, 254-257.

6 (a) V. Borsenberger, M. Kukwikila and S. Howorka, Org. Biomol. Chem., 2009, 7, 3826-3835; (b) C. T. Wirges, J. Timper, M. Fischler, A. S. Sologubenko, J. Mayer, U. Simon and T. Carell, Angew. Chem., Int. Ed., 2009, 48, 219-223; (c) V. Raindlová, R. Pohl, M. Šanda and M. Hocek, Angew. Chem., Int. Ed., 2010, 49, 1064-1066; (d) V. Raindlová, R. Pohl and M. Hocek, Chem.-Eur. J., 2012, 18, 4080-4087.

7 (a) H. Macíčková-Cahová and M. Hocek, Nucleic Acids Res., 2009, 37, 7612-7622; (b) H. Macíčková-Cahová, R. Pohl and M. Hocek, ChemBioChem, 2011, 12, 431-438; (c) P. Kielkowski, H. Macićková-Cahová, R. Pohl and M. Hocek, Angew. Chem., Int. Ed., 2011, 50, 8727-8730. 8 P. Ménová and M. Hocek, Chem. Commun., 2012, 48, 6921-6923.

9 P. Horáková, H. Macíčková-Cahová, H. Pivoňková, J. Špaček, L. Havran, M. Hocek and M. Fojta, Org. Biomol. Chem., 2011, 9, 1366-1371. 